\title{
LA LEY DEL DERECHO A LA CONSULTA PREVIA (29785) Y LA PROTECCIÓN DE LOS DERECHOS DE LOS PUEBLOS INDÍGENAS U ORIGINARIOS.2013
}

\author{
THE LAW OF THE RIGHT TO THE PRIOR CONSULTATION (29785) AND \\ THE PROTECTION OF THE RIGHTS OF THE INDIGENOUS OR ORIGINAL \\ PEOPLES.2013
}

PAMELA SILVANA SALCEDO ZEBALLOS 1

\section{Resumen}

El presente trabajo de investigación tiene el propósito de determinar si la Ley del Derecho a la Consulta Previa (29785) protege al Derecho de los Pueblos Indígenas u Originarios establecidos en el Convenio Internacional 169 de la OIT.Para ello se plantea las siguientes hipótesis: La Ley del Derecho a la Consulta Previa (29785) atenta y limita al Derecho de los Pueblos Indígenas $\mathrm{u}$ Originarios establecidos en el Convenio Internacional 169 de la OIT. La Investigación corresponde al análisis comparativo teórica, porque está orientada a contrastar la Ley con la aplicación a la solución de un problema, pues confronta la teoría con la realidad. También es un tipo de Investigación Socio Jurídica porque se estudian los hechos y relaciones de orden social reguladas por normas jurídicas y pretende determinar si la Ley del Derecho a la Consulta Previa (29785) protege al Derecho de los Pueblos Indígenas u Originarios establecidos en el Convenio Internacional 169 de la OIT. La recolección de información obtenida a través de técnicas del Cuestionario, Cédula de Entrevista y procesada a través de instrumentos de medición de variables de estudio. Finalizando la comprobación de las hipótesis de investigación. Palabras clave: Consulta previa, indígenas.

\section{Abstract}

The present research was conducted in order to determine whether the Law on the Right to Prior Consultation (29785) protects the right of Indigenous Peoples or Native established by the International Convention OIT.Para 169 it was established following hypothesis: The Law of the Right to Prior Consultation ( 29785 ) careful and limited to the Law of Indigenous or Native Peoples established by the International Convention 169 .The research is a form of applied research, it is focused on the application of knowledge to the solution of a problem, as the theory confronts reality. It is also a type of Socio Legal Research that examines the events and social relations regulated by legal norms and that is to determine whether the Law on the Right to Prior Consultation (29785) protects the right of Indigenous Peoples or Native established in the International 169 such purpose OIT.Para considered information obtained through the application of the questionnaire, and the Certificate of Interview, as instruments for measuring the study variables. The data were tabulated and analyzed using tables and graphs. After the phase of analysis and interpretation of the results was tested the hypothesis of our research.

Kay words: Prior consultation, indigenous.

\section{INTRODUCCIÓN}

El presente trabajo se pretende conocer si la Ley del Derecho a la Consulta Previa (29785) protege los Derecho de los Pueblos Indígenas $u$ Originarios sabiendo la situación vulnerable que se encuentran estos pueblos en nuestro país debido a que dichos pueblos son proclives a sufrir discriminación y no se encuentran protegidos sus derechos de una manera correcta. Es la obligación estatal de garantizar el derecho a la consulta, que no sólo se agota con la promulgación de una norma, va mucho más allá; siendo un paso importante pero no suficiente.

1 MAGISTER EN DERECHO CONSTITUCIONAL 
Cada vez que el ordenamiento constitucional reconoce un derecho fundamental, establece tácitamente tres obligaciones al Estado, desarrollar instituciones, procedimientos y asignar presupuesto para la real implementación del derecho reconocido.

En el Perú existen muchas comunidades campesinas que no están de acuerdo a que empresas mineras puedan explotar minerales, gas y petróleo del subsuelo de las tierras de territorios de las comunidades campesinas. La actitud de rechazo campesino comunero contra el abuso de empresas mineras tiene vigor en la actualidad. De ahí que el inciso $\mathrm{N}^{\circ} 2$. del Artículo 6 del Convenio 169 de la OIT, dice: “...con la finalidad de llegar a un acuerdo o lograr el consentimiento..." está contrapuesta a la posición de las comunidades campesinas de los andes y comunidades nativas de la amazonia del Perú. Lo establecido del Convenio está blindado con la mitad del segundo párrafo del Artículo 15 de la Ley 29785, cuando dice... "En caso de que no se alcance un acuerdo, corresponde a las entidades estatales adoptar todas las medidas que resulten necesarias para garantizar los derechos colectivos de los pueblos indígenas $u$ originarios y los derechos a la vida, integridad y pleno desarrollo". En el Perú se promulgó la Ley del Derecho a la Consulta Previa a los Pueblos Indígenas u Originarios, reconocido en el Convenio 169 de la Organización Internacional del Trabajo (OIT), conocido como LCPP, Ley 29785; la cual no cuenta con un reglamento para su aplicación, tampoco se sabe de cómo anda el problema de presupuesto y organización para la(s) "consulta(s) previa(s)".

Si las comunidades campesinas y comunidades nativas no llegan con el Estado del Perú a un acuerdo y consentimiento (aprobación) [Convenio 169 de la OIT] corresponde a las entidades estatales adoptar todas las medidas que resulten necesarias para garantizar los derechos colectivos de los pueblos indígenas $u$ originarios y los derechos a la vida, integridad y pleno desarrollo [LCPP]. Clavero, Bartolomé (2010) dice lo siguiente: "El derecho de consulta como expresión del autogobierno, y éste a su vez de la autodeterminación de los pueblos indígenas, reconoce su participación efectiva y libre en las esferas de decisión que les afecte...".

A fines de junio del año 2009, la Oficina de Control de la Magistratura (OCMA) impuso una histórica e inédita sanción contra tres magistrados de la Corte de Justicia de Lima. El motivo, desacatar una sentencia de la Corte Interamericana de Derechos Humanos (Corte IDH) (Ruíz.2011). "Uno de los principales problemas que existen en nuestro país es la falta de conciencia en los operadores del sistema de justicia..." (Berraondo.2006). A nivel constitucional, los derechos humanos reconocidos en el DIDH tienen como fundamento la dignidad humana. "Las normas relativas a los derechos y a las libertades que la Constitución reconoce se interpretan de conformidad con la Declaración Universal de Derechos Humanos y con los tratados y acuerdos internacionales sobre las mismas materias ratificados por el Perú" (Justiciaviva.org.pe.2011). Queda claro que los tratados internacionales de derechos humanos como los de los sistemas interamericano y universal de derechos humanos también forman parte del derecho nacional". (Clavero.1998). los tratados sobre derechos humanos, así como de las decisiones adoptadas por los tribunales internacionales sobre derechos humanos constituidos son tratados de los que el Perú es parte".(Chiacchiera.2009).

El Tribunal Constitucional (TC) ha establecido en reiterada y vinculante jurisprudencia que "Los tratados internacionales sobre derechos humanos de los que el Estado peruano es parte integran el ordenamiento jurídico. Los tratados sobre derechos humanos representan en tal sentido límites materiales de la propia potestad de reforma de la Constitución". (№ 00025-2005$\mathrm{PI} / \mathrm{TC}$ y No 00026-2005-PI/TC, acumulados, f.j. 34). (Anchante.2012).

El Estado reconoce y protege la pluralidad étnica y cultural de la Nación. Sin embargo, en un Estado constitucional de derecho, además de la Constitución deben tenerse en consideración las normas del bloque de constitucionalidad, entendido éste como el conjunto de normas "que se caracterizan por desarrollar y complementar los preceptos constitucionales relativos a [...] los derechos, deberes, cargas públicas y garantías básicas de los ciudadanos". (Ardito.2002). Cabe establecer que si bien los derechos reconocidos por el Convenio $\mathrm{N}^{\circ} 169$ de la OIT no están expresamente recogidos en la Constitución Política, éstos no sólo forman parte del derecho nacional sino que ostentan rango constitucional. "Con fecha 23 de agosto de 2011, el Congreso de la República aprobó por unanimidad la Ley $\mathrm{N}^{\circ}$ 29785, "Ley del Derecho a la Consulta Previa a los Pueblos Indígenas u Originarios, reconocidos 
en el Convenio № 169 de la Organización Internacional del Trabajo (OIT)". Dicha ley fue promulgada por el Presidente de la República con fecha 06 de septiembre de 2011 y se publicó en el diario oficial El Peruano el 7 de setiembre del 2011. Mediante la aprobación de la Ley de Consulta Previa, se reconocen los compromisos asumidos con el Convenio $169^{\circ}$ por ello, después de 16 años, y se constituye en el punto de partida para la solución de los conflictos entre el Estado y los pueblos indígenas, mediante el respeto y garantía de sus libertades". (Anaya.2005). A la luz de estos criterios de identificación, se puede afirmar que el Estado peruano no cuenta aún con una ley marco que englobe a todos los grupos colectivos y que permita identificar a los pueblos indígenas tal como lo hace el Convenio 169 de la OIT. Todo lo contrario: solamente se cuenta con legislación dispersa enfocada en grupos colectivos definidos de acuerdo con su ubicación geográfica. La realización de los procesos de consulta es una responsabilidad del Estado, y no de otras partes, tales como la empresa que busca obtener la concesión o el contrato de inversión (Tribunal Constitucional Peruano.2010). En conclusión se debe indicar que de no ser consultados los pueblos indígenas en forma previa, los derechos reclamados serán vulnerados; estando el Estado obligado a reparar los daños causados por la falta de validez del proceso consultivo.

La entidad competente para realizar la consulta es el Viceministerio de Interculturalidad del Ministerio de Cultura (Aranda.2010). A comienzos del mes de abril del año (2012), el Ministerio de Cultura emitió el reglamento de la Ley de Consulta Previa (Aylwin.2012). La consulta es un derecho que viene desde el

\section{OBJETIVOS}

Evaluar cómo se encuentra regulada la Ley de Consulta Previa (29785).

Analizar si se encuentran protegidos los Derechos de los Pueblos Indígenas u Originarios, según lo establecido en el Convenio Internacional 169 de la OIT y los Estándares de
Convenio 169, es un derecho ser consultados sobre cualquier medida administrativa legislativa que pueda afectar los derechos de los pueblos indígenas". (Landa.2011). AIDESEP representa a unas 1.800 comunidades indígenas (AIDESEP.2012).

Las organizaciones indígenas consideran que la consulta previa planteada tal cual en el Convenio 169 de la OIT puede generar grandes oportunidades para prevenir los conflictos sociales con los pueblos indígenas. Asimismo, resaltan que no están en desacuerdo con la inversión privada en sí misma, sino con las formas con las que se realizan los proyectos de inversión, sobre todo los extractivos, que son los que les afectan más".(Grupo Internacional de Trabajo Sobre Asuntos Indígenas. 2012).

El Estado peruano es uno de los países pioneros en entablar mecanismos legales e institucionales para llevar a cabo los procesos de la consulta previa. El Perú es uno de los primeros países en aplicar la Consulta Previa y se estaría avanzando en la prevención de los conflictos sociales que genera la inversión privada en los terrenos de los pueblos indígenas que muchas veces son afectados sin ser protegidos por el Estado.

"En relación con la importancia social y política de este conjunto de sentencias, consideramos que éste hace justicia a un sector de la población que históricamente fue marginado y excluido. $\mathrm{Si}$ sólo miramos, por ejemplo, la sentencia 000222009-PI/TC, de lejos la más importante, verificaremos que ella no sólo reconoce, sino que desarrolla el contenido constitucional protegido del derecho a la consulta previa de los pueblos indígenas (en adelante derecho a la consulta), contribuyendo de forma sustantiva con la implementación de este derecho". (Tribunal

\section{METODOLOGÍA}

Investigación de análisis comparativo teórico, socio. Diseño no experimental (ex post facto) Descriptivo - Explicativo.La muestra de estudio está conformada 31 profesionales en materia judicial: abogados litigantes, abogados de los Organismos Internacionales.

Identificar si es necesario la modificatoria de la Ley de Consulta Previa (29785) de acuerdo a lo establecido en el Convenio Internacional 169 de la OIT y los Estándares de los Organismos Internacionales.

empresas mineras (Southern, AngloAmerican, $\mathrm{CDH}$ y Minsur), defensores del Pueblo, operadores de justicia en defensoría del pueblo; y Fiscales Ambientales de la Región Tacna y Moquegua. 


\section{RESULTADOS}

El $87 \%$ de los abogados señalan que el Objeto de la Ley del Derecho a la Consulta Previa, establecido en el artículo $1^{\circ}$, no se encuentra regulada adecuadamente al señalar que se consultarán solo las afectaciones "directas, el $81 \%$ debería incluir toda afectación, no solo la "directa", y considerar toda fuente jurídica internacional, como la Declaración de Naciones Unidas sobre los Derechos de los Pueblos Indígenas, y no solo el Convenio $\mathrm{N}^{\circ} 169$ de la OIT; mientras que sólo el 19\% manifiesta lo contrario. El $77 \%$ consideran que el artículo 2 no se encuentra regulada adecuadamente al establecer sólo la protección de los derechos colectivos y el $85 \%$ señalan que el artículo 2 debe regular la protección de todo derecho indígena e invalidar todo acto no consultado a los pueblos. Asimismo, el 89\% de los abogados manifiestan que el artículo 10, el Estado debió garantizar la representatividad de las organizaciones, dado que es el deber del Estado de consultar a todos los pueblos afectados por la medida propuesta; el 81 considera que el artículo 15 no regula el consentimiento de consulta de los pueblos indígenas u originarios; y, el 19\% señala lo contrario. El 89\% opina que el artículo 15 debería precisar que la "decisión final" estatal está obligada a lograr el consentimiento cuando se consulta sobre la realización de megaproyectos, depósitos de relaves tóxicos, desplazamientos poblacionales o cuando se afecte la supervivencia; el $94 \%$ manifiestan que la ley garantiza el derecho a la identidad étnica de los pueblos indígenas u originarios. El $74 \%$ que la norma garantiza que el derecho de consulta sea de buena fe, con flexibilidad, transparente y su implementación sea previa a la toma de decisión.

El 96\% de los profesionales señala que la Ley del Derecho a la Consulta Previa no garantiza el derecho a Veto de los pueblos indígenas u originarios; el $77 \%$ afirma que no garantiza el derecho a la inclusión social de los pueblos indígenas u originarios; el 94\% manifiestan debe garantizar las oportunidades para la exigibilidad de los derechos de los pueblos indígenas $u$ originarios; el 90\% manifiestan que el Estado debe garantizar las condiciones institucionales para la plena vigencia de los derechos de los pueblos indígenas u originarios; el $89 \%$ señala que es imperativo, la apropiación de los derechos humanos como herramientas de legitimación por parte de los pueblos indígenas; ya que es imprescindible para la reconducción de los derechos de los pueblos indígenas a los derechos fundamentales y/o constitucionales;

\section{RESULTADOS DE ENTREVISTAS A MAGISTRADOS}

Respecto a si considera que el Objeto de la Ley del Derecho a la Consulta Previa debe considerar toda fuente jurídica internacional, así como lo establecido en el Convenio $N^{\circ} 169$ de la OIT, se tiene en respuesta que el objeto de la ley debe referirse estrictamente que pretende la norma en forma general, y en la exposición de motivos de incluirse todo marco normativo pertinente. Señalan ello porque el objeto es el primer artículo y debe señalar lo que regula la norma en su conjunto y además debe ser clara y de tan solo su lectura se debe desprender que abarca la norma que se está promulgando. Según la idea de que el artículo 2 de la Ley del Derecho a la Consulta Previa se encuentra regulada adecuadamente al establecer sólo la protección de los derechos colectivos, la opinión es que Sí. La razón es porque está bien regulada, porque lo que faltaba abordar normativamente era el derecho a la consulta y participación de los pueblos indígenas y originarios que le afectaban directamente, en ese marco es de suma importancia que se proteja sus derechos y se consulte y se les incluya antes de aprobar cualquier norma administrativa o legislativa relativa a sus derechos colectivos sobre su existencia física, identidad cultural, calidad de vida o desarrollo; así como los planes, programas y proyectos de desarrollo nacional y regional. Respecto a que si el el artículo 10 de la Ley del Derecho a la Consulta Previa, el Estado debió garantizar la representatividad de las organizaciones, dado que es el deber del Estado de consultar a todos los pueblos afectados por la medida propuesta, se tiene que están de acuerdo, porque el artículo $10^{\circ}$ establece los presupuestos que deben considerar para efectuar la consulta, esto es i) base de contenido de la medida propuesta; ii) el grado de relación directa con el pueblo indígena; y iii) el ámbito territorial de su alcance, los cuales garantizan que los pueblos indígenas $u$ originarias involucrados participen en la toma de decisiones sobre lo que les afecta. En el cuestionamiento si artículo 15 de la Ley del Derecho a la Consulta Previa, regula el 
consentimiento de consulta de los pueblos indígenas $\mathrm{u}$ originarios, la respuesta fue que no regula el consentimiento. Cuando no se pone de acuerdo, la decisión es arbitraria, correspondiendo a las entidades estatales adoptar las medidas necesarias para garantizar los derechos (ello queda establecido claramente en la segunda parte del segundo párrafo) y llevar adelante la aprobación de la norma o la ejecución de sus planes, programas o proyectos. Según el cuestionamiento de que la Ley del Derecho a la Consulta Previa garantiza la protección de los derechos de los pueblos indígenas $u$ originarios la respuesta fue que no en su totalidad. Si bien regulan el tema de participación y consulta, sin embargo, el poder Ejecutivo tiene la última palabra en la decisión en el caso de que los pueblos indígenas u originarios no están de acuerdo, creemos que lo grave es que reducen la consulta a una mera información y escucha de los involucrados, de acuerdo al Reglamento. No se puede garantizar la aplicación efectiva de la norma si es que todas las entidades públicas no han incluido en sus planes, programas 0 proyectos es te proceso como parte de sus respectivas políticas, cuyo trabajo está involucrado directamente a realizarlo con los pueblos indígenas u originarios.

\section{DISCUSIÓN}

A fin de comprobar la hipótesis planteada, se analizó la entrevista a los fiscales ambientales y defensores del pueblo de Tacna y Moquegua; observándose que más del $80 \%$ manifiestan que La Ley de Consulta Previa (29785) se encuentra regulada inadecuadamente, debido al incumplimiento del objeto de la Ley, el derecho a la consulta e identificación de los pueblos indígenas a ser consultados. El $85 \%$ de los abogados manifiestan que los Derechos de los Pueblos Indígenas se encuentran desprotegidos en función a lo establecido en el Convenio Internacional de la OIT y los Estándares de los Organismos Internacionales, ya que no se ejerce el derecho a la identidad étnica y el derecho de Consulta, la cual debe ser de buena fe, que admita flexibilidad, el alcanzar un acuerdo razonable y que prime la transparencia y la implementación previa a la toma de decisión. El $80 \%$ manifiestan que es necesario la modificatoria de la Ley de Consulta Previa (29785) de acuerdo a lo establecido en el Convenio Internacional 169 de la OIT y los Estándares de los Organismos Internacionales.

La Ley de Consulta Previa (29785) sí limita el Derechos de los Pueblos Indígenas u Originarios, por su incoherencia con lo establecido en el Convenio Internacional 169 de la OIT.2013 y se encuentra regulada inadecuadamente, lo que origina no alcanzar acuerdos razonables. Los derechos de los Pueblos Indígenas u Originarios se encuentran desprotegidos por la Ley por su incoherencia con el Convenio Internacional 169 de la OIT y los Estándares de los Organismos Internacionales sobre Derechos Humanos. Debería modificarse en congruencia con lo presentado por estos organismos. El Estado debe manifestar su preocupación por los Derechos de los Pueblos Indígenas en función a lo establecido en el Convenio Internacional de la OIT y los Estándares de los Organismos Internacionales, con el pleno ejercicio del derecho a la identidad étnica y el derecho de Consulta, la cual debe ser de buena fe, que admita flexibilidad, el alcanzar un acuerdo razonable y que prime la transparencia y la implementación previa a la toma de decisión. Para ello es preciso conformar en el nivel nacional una mesa de diálogo entre las organizaciones indígenas y el Estado que funcione de manera permanente e institucionalizada para ir definiendo de manera progresiva la aplicación del marco jurídico vigente. El Congreso debe presentar una propuesta de Ley con la modificatoria de la Ley de Consulta Previa (29785) de acuerdo a lo establecido en el Convenio Internacional 169 de la OIT y los Estándares de los Organismos Internacionales; considerando los siguientes aspectos:

Artículo 1.- Señala que se consultarán solo las afectaciones "directas", cuando debería incluir TODA afectación, no solo la "directa", y considerar toda fuente jurídica internacional, como la Declaración de Naciones Unidas sobre los Derechos de los Pueblos Indígenas, y no solo el Convenio $N^{\circ} 169$ de la OIT.

Artículo 2.- Solo protege los derechos colectivos, debiendo proteger TODO derecho indígena e INVALIDAR todo acto no consultado a los pueblos.

Artículo 15.- No regula el consentimiento. Debería precisar que la "decisión final" estatal está obligada a lograr el consentimiento cuando se consulta sobre la realización de 
megaproyectos, depósitos de relaves tóxicos, desplazamientos poblacionales o cuando se afecte la supervivencia; y en un segundo momento de diálogo; a no afectar el derecho a un medio ambiente equilibrado.

La Ley del Derecho a la Consulta Previa (29785) debe contemplar el derecho a Veto, el Derecho a la inclusión social, el Derecho a la identidad étnica y el derecho de Consulta a fin de no atentar y limitar el Derecho de los Pueblos Indígenas u Originarios establecidos en el Convenio Internacional 169 de la OIT. Asimismo, el Estado debe promover la revalorización de la identidad indígena tanto al interior delos mismos pueblos indígenas como en la sociedad en general, para conocer y comprender los aportes que pueden hacer al desarrollo del país.

Presentado: Marzo 2017

Aceptado: Junio 2017

\section{REFERENCIAS BIBLIOGRÁFICAS}

Anaya, J. (2005). Los Pueblos Indígenas en el Derecho Internacional.Madrid: Ed. Trotta.

Aranda, M. (2010). Los Desafíos de la implementación de la Consulta Previa en el Perú. http://biblio.juridicas.unam.mx.México.

Asociación Interétnica de Desarrollo de la Selva Peruana (AIDESEP) y otros. Perú.Informe alternativo. (2012). Sobre el cumplimiento del Convenio 169 de la OIT. Lima:AIDESEP.

Aylwin, J. (2012). El derecho de los pueblos indígenas a la tierra y al territorio en América Latina: Antecedentes históricos y tendencias actuales. Universidad de la Frontera. Temuco.

Berraondo, M. (2008). La Declaración sobre los derechos de los pueblos indígenas. Punto y seguido, Alternativa.Barcelona.

Chiacchiera, P. (2009). La cuestión indígena. Análisis de la jurisprudencia de la C.S.J.N. Córdoba: Advocatus.

Clavero, B. (1998). Derechos de los pueblos indígenas. Servicio Central de Publicaciones del Gobierno Vasco.Vitoria-Gasteiz.

Comisión Interamericana de Derechos Humanos. (2000). La situación de los derechos humanos de los indígenas en las Américas. O.E.A. Washington D.C.

Comisión Interamericana de Derechos Humanos. (2001). Fuentes en el derecho internacional y nacional del proyecto de declaración americana sobre los derechos de los pueblos indígenas.O.E.A. Washington D.C.

Corte Interamericana de Derechos Humanos (2010). Derechos de los pueblos indígenas y tribales sobre sus tierras ancestrales y recursos naturales: Normas y Jurisprudencia del Sistema Interamericano de Derechos Humanos. OEA/ Ser.L/V/II. Doc. 56/09, aprobado el 30 de diciembre de 2009 y publicado en 2010.

DPLF (2010). El Derecho a la Consulta Previa, libre e informada de los pueblos indígenas.

Grupo Internacional de Trabajo Sobre Asuntos Indígenas. (2012). El mundo indígena. Copenhague: IWGIA.

Ruiz, J. (2011). La implementación del derecho a la consulta previa de los pueblos indígenas. Una mirada constitucional. Lima.

Sentencia del Tribunal Constitucional. (2009). / EXP. N. ${ }^{\circ} 00022-2009-\mathrm{PI} / \mathrm{TC}$

LIMA.GONZALO TUANAMA TUANAMA Y MÁS DE 5000 CIUDADANOS.

Sentencia del Tribunal Constitucional. (2010). / EXP. N. ${ }^{\circ}$ 00024-2009-PI

Tribunal Constitucional Peruano.La Ley de Consulta Previa. 